\title{
Der Einfluss der Kälteapplikation auf die Pulsfrequenz von Hunden mit ausgeschalteten extrakardialen Herznerven. $\S$
}

\author{
Von \\ Masao Wada. \\ (和由正男) \\ (Aus dem Physiologischen Institut von Prof. I. Satake, \\ Tohoku Reichsuniversität, Sendai.)
}

Vor etwa zehn Jahren untersuchten Cannon und seine Mitarbeiter ${ }^{13}$ den Kälteeinfluss auf Katzen, bei denen auf operativem Wege die extrakardialen Herznerven vollständig ausgeschaltet worden waren und konnten dabei eine recht ansehnliche Beschleunigung des Herzschlages feststellen. Wenn ausserdem noch die rechte Nebenniere fortgenommen und die linke völlig entnervt worden war, hatte eine Kälteapplikation vom selben Grade an demselben Tier diesmal entweder nur eine geringfügige Beschleunigung oder sogar eine Verminderung des Pulses zur Folge. Die Verfasser glauben mit diesen Versuchen den Beweis dafür erbracht zu haben, dass durch die Abkühlung des ganzen Tieres die Nebennieren zu vermehrter Epinephrinabsonderung anregt werden, wovon auch bei $\mathrm{H}$ artm a n et al..$^{2}$ auf Grund ihrer Beobachtungen an Katzen mit völlig entnervten Pupillen die Rede war.

Angeregt durch diese Arbeiten nahm bald darauf Saito ${ }^{3)}$ im hiesigen Institute Abkühlungsversuche an einer Reihe nicht narkotisierter und nicht gefesselter Hunde vor, aber mit durchaus negativem Erfolg. Das Nebennierenblut wurde hierbei direkt aus der Nebennie-

§ Kurz mitgeteilt auf dem Japanischen Physiologen-Kongress, 1934, 4. A pril: Jap. J. of Med. Sci., Biophysics, 1934, 3, 222.

1) Cann oul und Querid o, Proc. Nat. Acad. Sci. U.S.A., 1924, 10, 245 ; Cann on, Querido, Britton und Bright, Am. J. of Physiol., 19 (26-) 27, 79, 466.

2) Hartman, McCordock und Loder, Am. J. of Physiol., 1923, 64, 1,; Hartman und $\mathrm{H}$ artman, Ebenda, 1923, 65, 612.

3) Saito, Tohoku J. of Exp. Med., 1928, 6, 544. 
renvene schmerzfrei vom Rücken her entnommen und sein Epinephringehalt mittelst des Kaninchendarmstückverfahrens abgeschätzt.

Welche Versuchsbedingungen sind für diese Diskrepanz zwischen den Resultaten von Cannon et al. und Saito verantwortlich zu machen? Was die Verschiedenheiten der Bedingungen der beiden zu vergleichenden Versuchsreihen anbetrifft, so ist zunächst zu bemerken, dass zwei verschiedene Tierarten, Katze und Hund, verwendet wurden. Die Katze ist bekanntermassen sehr empfindlich gegen Kälte, während derHund sich sehr widerstandfähig gegen sie verhielt. $Z$ weitens wurden zur Applikation der Kälte verschiedene Verfahren benutzt. Die Katzen von Cannon wurden entweder der kalten Luft ausgesetzt, oder es wurde ihnen eine gewisse Menge eiskaltes Wasser durch den Mund in den Magen eingeführt. Saito dagegen bedeckte den Hund mit einem doppelwandigen Gummimantel und liess durch dessen Zwischenlumen eiskaltes Wasser hindurchfliessen. In einigen Fällen liess er auch den Hund an der Bauchdecke mit dem Eisblock unmittelbar in Berührung kommen. Hartman schliesslich tauchte die Katzten ins Eiswasser. Jedenfalls war der Abfall der Körpertemperatur jedoch in den Experimenten Cannons und Saitos nie besonders gross.

$\mathrm{Zu}$ diesen beiden Verschiedenheiten der Versuchsbedingungen kommt noch eine dritte hinzu. Cannon und Saito benutzten nämlich zur Abschätzung der Geschwindigkeit der Epinephrinabgabe verschiedene Verfahren. Diese Verschiedenheit soll aber nicht für die Diskrepanz zwischen den beiden Versuchsresultaten verantwortlich gemacht werden, falls jede der beiden Methoden einwandfrei ist.

Um nun eine Lösung der Frage in die Wege zu leiten, wurden die folgenden Versuchsreihen von uns angestellt.

(1) Die Sekretionsgeschwindigkeit des Epinephrins aus der Nebenniere wurde mittels des Verfahrens von Satake et al., wie im hiesigen Laboratorium übrich, gemessen. Erstens wurde einem Hunde nach $\mathrm{C}$ annons Beispiel eiskaltes Wasser in den Magen eingeführt, und zwar nicht wie in seinen Versuchen durch den Mund, sondern durch eine vorher hergestellte Magenfistel, da die Einführung durch den Mund schon an und für sich sehr beschleunigend auf die Pulszahl einwirken kann, wie Cannon es auch selbst zugab. Zweitens wurde der Hund solange ins eiskalte Wasser getaucht bis seine Analtemperatur auf $30^{\circ} \mathrm{C}$ gesunken war, eine Temperatur, die von den Forschern des Kältediabetes vom Alters her angewendet wurde. Die Daten dieser Versuche wurden bereits in einer vorangegangenen Mitteilung: 
veröffentlicht. Eine doppelt so grosse Wärmeschuld wie die, mit der Ca n non seine Katzen belastet hatte, war fast völlig ausserstande, eine unverkennbare Hypersekretion des Epineplrins hervorzurufen, während eine starke Abkühlung im Eiswasser eine hochgradige Hypersekretion zur Folge hatte.

An Katzen wurden die Untersuchungen dieser Art nicht ausgeführt, da Verf. den Körper dieses Tieres, insbesondere die Nebennierenvene, für zu klein hielt.

(2) Parallel zu den oben geschilderten Experimenten, wurden Versuche an Hunden mit völlig entnervtem Herzen angestellt. Diese Reihe von Experimenten wurde schon im Jahre 1929 begonnen, und der Operationserfolg der Entnervung wurde stets nach dem Tode des Tieres freundlicherweise von dem Pathologen, Herrn Prof. Dr. S. Nasu, kontrolliert.

Hund Nr. 21 (1932) war der erste, bei dem die Entnervung sich als tadellos ausgeführt erwies.

Während dieser Zeit wurde eine Reihe von Mitteilungen über die mit völlig entnervtem Herzen gewonnenen Ergebnisse aus dem Bostoner physiologischen Laboratorium veröffentlicht und zwar wurden die übrigen Faktoren, die den Rhythmus des entnervten Herzens irgendwie zu beeinträchtigen vermögen, entdeckt; schliesslich wurde ,flat" Präparat, d.h. die Katze, deren Herzschlagszahl von den äusseren Agentien, wie psychische Aufregung etc., nie beeinflussbar ist, ${ }^{52}$ hergestellt. Endlich wurde noch die Existenz einer Substanz entdeckt, die sich an der Sympathikusendigung bei deren Erregung bildet und absondert, um auf dem Blutwege auf das Herz beschleunigend einzuwirken, wie es das Epinephrin aus den Nebennieren tut. Cannon ${ }^{6)}$ nennt diese Substanz Sympathin. Bei sympathischen Erregungen wie psychischer Aufregung wird soviel Sympathin in die Blutbahn geschickt, dass das entnervte Herz eine beträchtliclie Akzeleration erfährt.

Es war nun wünschenswert, mit der so fortgeschrittenen Technik den Abkühlungsversuch noch einmal zu wiederholen, da er in einer Zeitperiode angestellt worden war, in der es noch viel mangelhaftes in der Technik und ebenso in der Kenntnis der das entnervte Herz in situ beeinflussenden Faktoren gab, umsomehr als wohl keine wesentlichen Differenzen in der Grösse der Pulsakzeleration vor und nach der Operation der Nebennieren übrig bleiben, wenn man die Tatsache

4) Wa da, Seo und A be, Tohoku J, of Exp. Med., 1935, 26, 381 .

5) Newton, Zwemer und Cannon, Am.J. of Physiol., 1931, 96, 377.

6) Cannon und Bacq, Ebenda, 1931, 96, 392. 
berücksichtigt, dass die Grösse der Pulsvermehrung durch Fesselung derKatze nach der Ausschaltung der Nebennieren markfunktion in der Publikation vom Jahre 1931 deutlich grösser war im Vergleich zu der des Jahres 1927, bei der der Abkühlungsversuch gleichzeitig mitgeteilt worden war. Ferner kommt dazu, dass eine psychische Aufregung wie die bei der Fesselung des Tieres das entnervte Herz anscheinend weit stärker beeinflusst als die Abkühlung, und die Einführung der Sonde durch den Mund eine grosse Aufregung seitens ảer Katze, wie von Cannon angegeben wurde, zu Folge hat.

Vorf. hält es daher für angebracht, seine diesbezüglichen Es fahrungen an Hunden mit völlig entnervtem Herzen hier mitzuteilen.

In thbereinstimmung mit den Bostoner Physiologen war der Autor imstande eine unverkembare Beschleunigung des entnervten Herzens durch Einführung von eiskaltem Wasser in den Magen durch die Magenfistel zu beobachten, jedoch traten dieselben Erscheinungen, im qualitativen sowie im quantitativen Sinne, ebenfalls nach der Demedullation der Nebennieren auf, so dass eigentlich nichts von der Pulsbeschleunigung übrig bleibt, was eine Beteiligung der velmehrten Epinephrinsekretion an dieser beweisen könnte.

Der Darstellung meiner Versuchsdaten soll eine kurze Skizze der Literatur betreffs des Gebrauches des entnerrten Herzens in situ als Indikator der Geschwindigkeit, mit der das Epinephrin aus der Nebenniere ausfliesst, folgen. da seit der Darstellung von $K_{\text {od a mai }}$ ) im Jahre 1923 noch viele wichtige Publikationen hierüber erschienen sind.

1912 erkannte A n r e ${ }^{8)}$ in Versuchen mit narkotisierten Katzen, dass eine faradische Reizung des peripheren Endes des Splanchnicus, im Einklang mit früheren Untersuchern, eine erhebliche Pulsbeschleunigung des durch Vagotomie und Exstirpation der beiden Ganglia stellata entnervten Herzens erzielt; er sah ferner, dass sie verschwand, oder viel geringer war sowohl nach Abklem. mung der Nebennierenvenen als auch nach Entfernung der Nebennieren, was eine Mehrabgabe des Epinephrins beweist. Sechs Jahre später berichteten jedoch $S$ te wart und Rog of $f^{9}$ im Gegensatz zu den Angaben von A v re p, dass. eine Reizung des zentralen Stumpfes eines Nervus ischiadicus sowie des peripheren Endes des Splanchnicus eine auffallende Schlagbeschleunigung des entnervten Katzenherzens selbst nach dem Abklemmen der Nebennierenvenen

7) Kod a ma, Tohoku J. of Exp. Med., 1923, 4, 166.

8) Anrep, J. of Physiol., 1912, 45, 307. Die Angabe von Anrep im nächsten Jahre wurde von Levg anscheinend bestätigt, d. h. Reizung des peripheren Endes des Splanchnikus hat eine Beschleunigung des vom Ganglia stellata befreiteten Herzens in situ einer Katze, zur Folge, solange die Nebenniere selbst intakt bleibt. (Levs, Heart, 19(12-)13, 4. 343.)

9) Stewart und Rog of $f$, Am. J. of Physiol., 1918, 46, 96 f. 
herbeiführt. Dieses Phänomen wird nach ihrer Ansicht durch bessere Blutdurchströmung im Coronargefäss bedingt. Auf der andern Seite aber glaubte $\mathrm{Cann} 0 \mathrm{n}^{10)}$ durch dieses Präparat einen Beweis für die Richtigkeit seiner früheren Anschaumng gefunden zu haben, nach der die Nebemieren bei afferenter Reizung und bei Asphyxie mehr Epinephrin absondern. Das entnervte Herz der mit Urethan narkotisierten Katze schlug bei der Reizung des zentralen Stumpfes des Ischiadicus, z. B. um 50 Schläge pro Minute schneller. Diese reflektorische Pulsbeschleunigung fehlte nach der Nebennierenentfernung. Eine Wiederholung dieser Versuche wurde bald von Stewart und Rog of $\mathrm{f}^{11}$ ) mit völlig anderen Ergebnissen, als $\mathrm{C}$ a $\mathrm{n}$ n on et al. sie gefunden hatten, ausgeführt. Sie wandten deshalb ein, dass unter den genannten Bedingungen die Pulsbeschleunigung vorwiegend die Folge der Zunahme der in der Zeiteinheit durch die Coronargefässe fliessenden Epinephrinmenge darstellt, infolge der durch die Blutdrucksteigerung bedingten ,Wiederverteilung" des Blntes. Aus der Tatsache, dass in einem Fall die Ischiadicusreizung nach beiderseitiger Splanchnikotomie trotz der Blutdruckerhöhung keine deutliche Schlagfrequenzvermehrung bewirkte, nahmen sie an, dass das Ausbleiben der Herzreaktion auf den Ausfall der Vermittlung irgendeines unbekannten, aus der Leber oder aus dem Darm reflektorisch abgegebenen Stoffes zurückzuführen sei. Canuon und sein Mitarbeiter ${ }^{12}>$ kameu dann nachträglich dazu, den Befund der Gegner teilweise zu bestätigen, indem sie eine Beschleunigung des entnervten Her. zens, weun auch von viel geringerem Grade als bei intakten Nebennieren, durch Reizung des peripheren Endes des Splanchnicus oder des zentralen des Brachialis beobachteten, selbst wenn die Nebennieren zuvor herausgenommen worden waren; diese trat aber nicht mehr auf, oder verschwand sogar, wenn die $V$. cava inferior und die V. portae unterhalb der Leber abgebunden oder die Lebermerven durchschnitten worden waren. Vom Agenz aus der Leber, das auf das entnervte Herz beschleunigend einwirkt, haben wir einige weitere Angaben. ${ }^{13}$ ) Die widersprechenden Resultate über den Einfluss der Splanchnicusreizung auf das entnervte Katzenherz in situ bei nicht vorhandenen Nebennieren sollen nach diesen Autoren damit zusammenhängen, dass die Reizung der Lebernerven einen rascheron Schalg des denervierten Herzens zwar nicht bei hungernden Tieren, wohl aber bei fleischgefütterten Tieren erzielen kann. Auch Searles ${ }^{14}$ gab an, dass die residuelle Schlagfrequenzvermehrung des entnervten Herzens nach Abdrosselung des Abflusses der Nebennieren nur bei nicht fastenden Hunden vorhanden ist. Bei dieser Sachlage versuchte Cannon zusammen mit Rapport, seine Ansicht, dass das entnervte Herz einen exquisiten Indikator der Epinephrinabgabe darstelle, durch zweierlei Experimente

10) Cannon, Seience, 1917, 45, 463; Am. J. of Physiol., 1919, 50, 399.

11) Stewart und Rog off, Ebenda, 1920, 52, 304 u. 521.

12) Cannon und Rapport, Ebenda, 1921, 58, 308.

13) Cannon und Uridil, Am. J, of Physiol., 1922, 58, 353; Cannon, Uridil und Griffith, Endocr, 1921, 5, 729; Cannon und Griffith, Am. J. of Physiol., 1922, 59, 480 (Katze); A s her, Pflügers Arch., 1925, 209, 605; Schweiz. m. W., 1926, 921 (Frosch); Rosenbleuth und Phillips, Am. J. of Physiol, 1932, 102, 332 (Hund).

14) Searles, Am. J. of Physiol., 1923, 66, 408. 
zu erhärten. In der ersten Versuchsgruppe wiesen sie nach, dass bei ,reduzierten" ebenso wie bei ,unreduzierten" Tieren dieselbe Beschleunigung des entnervten Herzeus bei afferenter Reizung erzielt werden kann. Bei den sogenannten ,reduzierten“ 'Tieren waren beide Carotiden, beide Brachiales, beide Nierenarterien und die Aorta unterhalb der Nieren abgebunden und die Mesenterialnerven durchschmitten, um dadurch das Gebiet der reflektorisch beeinflussbaren Blutgefässe auf ein Minimum zu bringen, und von den Abdominalorganen die Nebennieren allein durch die Splanchnicusnerven innerviert sein zu lassen. In der zweiten Versuchsgruppe, wo nach der Entfernung der Nebennieren mit oder ohne Durchschneidung der Lebernerven eine gleichmässige Dauerinfusion des Adrenalins in der Menge von 0.0007 bis $0.0008 \mathrm{mg}$ pro $\mathrm{kg}$ pro Minute in die Vena femoralis gemacht worden war, um die kontinuierljche, unveränderliche Nebemierensekretion zu ersetzen, wurde die typische Pulsbeschleunigung nach afferenter Reizung nicht mehr beobachtet, whihrend die Blutdrucksteigerung die gleiche war wie vor der Nebennierenentfernung. Als weiteres Beweismaterial dafür, dass die Frequenzsteigerung des entnerten Herzens, die die afferente Reizung oder Asphyxie herbeiführt, nur eine direkte Folge der Epinephrinmehrsekretion ist, machten Cannon und CarrascoFormiguera ${ }^{15}$ folgende Versuche. Sie beobachteten, dass das entnervte Katzenherz auf eine afferente Reizung (des Nervus brachialis) oder Asphyxie nicht mit typischer Frequenzvermehrung antwortete, wenn die Vv. lumbales lateralseits der Nebennieren, alle benachbarten Venen, die Nierengefässe und die Vena cava unterhalb der Nieren abgebunden waren und ferner die Vena cava dicht unterhalb der Leber abgeklemmt worden war; nach dem Wegnehmen der Klemme war dagegen die reflektorische Herzbeschleunigung wieder gut zu erzielen. Das Intervall zwischen dem Anfang der afferenten Reizung und dem Beginn der Reaktion des Herzens entspricht nach ihnen annäherungsweise der Zeit, die vergeht, bis das in die Femoralvene injizierte Adrenalin seine herzbeschleunigende Wirkung entfaltet. Später gelang es Cannon und seinen Mitarbeitern, ${ }^{16)}$ die Katzen am Leben zu erhalten, nachdem sie ihnen das Herz durch Exstirpation der beiden Ganglia stellata und mittels Durchtrennung des rechten Vagus unterhalb des Recurrens und aller von dem linken Vagus abzweigenden Äste bis zum unteren Oesophagus, vom Zentralnervensystem abgetrennt hatte. Dabei war es für Cannon überraschend, dass bei den so operierten Tieren eine beträchtliche Pulsbeschleunigung um 79-84 Schläge pro Minute bei Gemütserregungen, verbunden mit grossen körperlichen Anstrengungen, in Erscheinung trat, auch wenn die bekaunten humoralen Einflüsse durch Entnervung der Schilddrüse und Leber, und durch Herausnahme der einen Nebenniere und Entnervung oder Markentfernung der anderen ausgeschaltet worden waren. Nach Cannon ist diese Beschleunigung nicht auf eine Regeneration der abgetrennten Herznerven, nicht auf Blutdrucksteigerung, und auch nicht auf Produkte des Muskelstoff wechsels zurückzuführen. Auch durch Ausschaltung der humoralen Einflüsse, nämlich der Schilddrüse, der Hypophyse

15) Cannon und Carrasco-Formiguera, Am. J. of Physiol, 1922, 61, 215.

16) Cannon, Lewis und Britton, Ebenda, 1926, 77, 326. 
oder des akzessorischen chromaffinen Gewebes wurde unter diesen Bedingungen das Auftreten der Herzbeschleunigung $z$ war nicht gehemmt, wohl aber war sie nach Entfernung der beiden oberen thorakalen sympatischen Grenzstränge stark reduziert. Die letztgenaunte Tatsache weist auf die Beteiligung der Herzäste, die von den oberen thorakalen sympathischen Grenzsträngen, abzweigen, am Zustandekommen der Herzbeschleunigung bei nicht narkotisierten Tieren hin. Demnach scheint es notwendig zu sein, ausser der Ganglia stellata und der Nervi vagi die Grenzstränge im oberen Thorakalgebiete auszurotten, wenn man eine vollständige Denervierung des Herzens erhalten will. Hier sei darauf hingewiesen, dass die eingangs erwähnten Ablühlungsversuchen der Bostoner Physiologen gerade an den in dieser Weise hergestellten Präparaten ausgeführt wurden.

Interessante neue Beiträge zu diesen Fragen wurden weiter aus dem $\mathrm{C}$ a nnonschen Laboratorium geliefert. Cannon und seine Mitarbeiter ${ }^{5)}$ stellten fest, dass die Schlagzahl des völlig entnervten Herzens bei nicht narkotisierten, am Leben erhaltenen Katzen durch eine 1 Minute anhaltende Fesselung der Tiere in der Rückenlage meist um 30 Schläge pro Minute gesteigert werden konnte, selbst wenn der Einfluss der Epinephrinsekretion durch Herausnahme einer Nebenniere und Denervierung oder Markentfernung der anderen unterdrückt worden war, ausser der Ausschaltung der anderen bekannten humoralen Agenzien (Leber und Schilddrüse). Diese „,rätselhafte" von Cannon nicht erklärbare Herzfrequenzvermehrung erreichte in der Regel nach etwa 3 Minuten ihr Maximum und liess dann langsam nach. Diese Beschlennigung wurde durch irgend welche operative Eingriffe abgeschwächt und trat bei tief narkotisierten Tieren nicht auf. Wurden alle Abschnitte des Sympathicus mit Ausnahme der kurzen Grenzstränge im unteren Thorakalgebiete, vom Rückenmarke abgetrennt, so kam die Herzantwort nur noch nach einer Aufregung zustande, aber nach Entferuung der zurückgelassenen Abschnitte trat sie nicht mehr auf. Cannon und Bac q zeigten weiterhin, ${ }^{6}$ ) dass bei narkotisierten Katzen mit denerviertem Herzen, deren Nebennieren und Leber inaktiviert und deren Rückenmark im mittleren Brustmark durchtrennt worden war, eine faradische Reizung der zu der glatten Muskulatur der Schwanzhaare führenden sympathischen Nervenfasern eine allmähliche Zunahme der Pulsfrequenz bewirkt, die ihr Maximum in zwei oder drei Minuten erreichte und dann graduell zum Ausgangs wert zurückkehrte. Aus der Tatsache, dass die unter dieser Bedingung feststellbare Herzbeschleunigung erheblich abgeschwächt oder aufgehoben wurde, wenn der Blutumlauf im betreffenden Gebiete während der Reizung unterbrochen oder die betreffende Partie aus dem allgemeinen Kreislauf ausgeschaltet worden war, glauben sie, dass ein dem Epinephrin ahnlicher Stoff, das Sympathin, aus der vom Sympathicus innervierten glatten Muskulatur an den Kreislauf abgegeben wird, der am Zustandekommen der Herzbeschleunigung beteiligt sein soll.

Nach den oben erwähnten Versuchen scheint im Dauerpräparat des entnervten Herzens eine nicht ganz einwandfreie Methode zum Nachweis der Epinephrinselrretion gegeben zu sein, insofern als alle von den sympathischen Zentren ausgehenden, sympathinproduzierenden Einflüsse mit Auswahme der 
Nebennieren nicht ausgeschaltet werden. Es fragt sich nun, ob bei der Abkühlung des Tieres die Sympathinabsonderung, nicht wie bei der Gemütserregung, zu gering ist, um an der dabei hervorgerufenen, charakteristischen Pulsfrequenzsteigerung des entnervten Herzens nennenswerten Anteil zu haben, weil bei den Versuchen von Cannon und seinen Mitarbeitern im Jahre 1927 diese Frequenzsteigerung nach der Inaktivierung des Nebennierenmarkes, wie eingangs erwähnt, nur unbedeutend war oder sogar nicht selten eher eine Verlangsamung erfolgte, trotzdem die sympathischen Grenzstränge mit Ausnahme der oberen thorakalen Segmente intakt gelassen worden waren.

\section{Methodik.}

Zur Herstellung der Dauerpräparate des entnervten Herzens in situ bei Hunden wurden junge, kleine, kräftige Tiere sowohl Männchen wie auch Weibchen ausgewählt. Das operative Verfahren, das an unseren Tieren ausgeführt wurde, stimmt im Prinzip mit den im Jahre 1926 von Cann on, Lew is und Britton beschriebenen und kurz danach bei den oben erwähnten Abkühlungsversuchen an Katzen angewandten, überein.

Die dauernde Entnervung desHerzens wurde durch folgende Eingriffe erreicht:

1. durch beiderseitige Herausnahme der Ganglia stellata mit den oberen thorakalen sympathischen Grenzsträngen,* 2. durch linksseitige Durchtrennung aller vom Vagus sowie vom unteren Halsganglion abzweigenden Äste bis zum Oesophagus, und 3. durch rechtsseitige Durchschneidung des Vagosympathicus kurz oberhalb des unteren Halsganglions.

Die Art der Entnervung ist aus Abb. 1 ersichtlich, in der die Herznerven des Hundes schematisch dargestellt sind. Die punktierten Linien zeigen die zu excidierenden oder herauszunehmenden Teile an.

Da unser Verfahren mit dem von Cann on und seinen Mitarbeitern in den Details nicht völlig übereinstimmt, fühle ich mich berechtigt, es etwas ausführlicher hier anzugeben. Wir gingen also im einzelnen auf folgende Weise vor:

Die Hunde wurden durch eine Subcutaneinspritzung einer 2\%igen Morphinlösung in einer Menge von etwa $0,5 \mathrm{ccm}$ pro $\mathrm{kg}$ narkotisiert. Sobald die Narkose tief genug war, wurden die Tiere auf dem Rücken liegend festge-

* Die sich von den oberen thorakalen Grenzsträngen zum Herzen herabziehenden Nervenäste beim Hunde sind bei folgenden Autoren angegeben. I o nes cu und En achescu, Ztschr.f. Anat. u. Entwickl., 1928, 85, 476; Schurawle w, Ebenda, 1928, 86, 655; I onescu, Bernard, Iliescu und Enachescu, Pflügers Arch., 1928, 219, 47 ; I onescu, Enachescu und Teite1, Klin. Wschr., 1928, 7 Jg., 991. 
Abb. 1.

v.s.d.= vagosymp. dex. v.s.s. = vagosymp. $\sin$. g. c.i.d.= gangl. cerv. g.c.i.s.=gangl. cerv. inf. dex.

g. st.d. = gangl. stellat. dex.

t.s.d. $=$ trunc. symp. dex.

v. $d$. = ventricul. cord. dex.

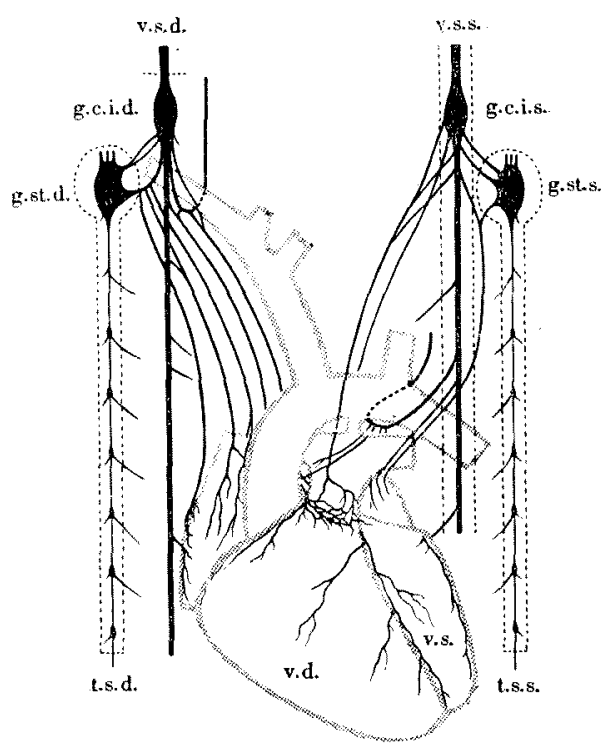

bunden. Während der darauf folgenden $\ddot{A}$ therinhalation wurde dem Tier das Maul aufgehalten und ein vorher sterilisierter $30 \mathrm{~cm}$ langer Gummikatheter in die Trachea so tief eingeführt, bis sich dessen schräg geschnittene Spitze einige Zentimeter oberhalb der Bifurcation befand. Das andere Ende des Katheters wurde durch ein in der Mitte eines hölzernen, spindelförmigen Stäbchens befindliches Loch geleitet und durch einen Gummischlauch mit einem durch einen Elektromotor in Bewegung gesetzten Blasebalg verbunden. Das Stäbchen wurde hinter den Eckzähnen festgehalten, indem man einen Strick um das Maul band. Nun wurde der Katheter mit einem Faden am Stäbchen befestigt. Die Tiefe der Narkose wurde nach Belieben durch die Einschaltung einer mit einer speziellen Vorrichtung versehenen $\ddot{A}$ thernarkoseflasche in die Luftleitung reguliert.

Um die linksseitige Entnervung des Herzens bequem ausführen zu können, wurde die vierte Rippe an dieser Seite nach Ablösen des Periostes mit Messer und Elevatorium freigelegt und ein mindestens $5 \mathrm{~cm}$ langes Stück derselben dorsalwärts von der Übergangsstelle zum Rippenknorpel mit einer Hohlmeisselzange entfernt. In der Mitte des hinteren Periostes wurde ein kleiner Einschnitt unter Schonung der Lunge gemacht. In diese öffnung wurden die unteren Branchen von zwei Gefässklemmen eingeschoben; damit wurde der hintere Periost an irgendeiner Stelle, vom Loch aus mit den darunterliegenden Geweben seitlich gefasst und zwischen den beiden Gefässklemmen mit einer stumpfen Schere abgeschnitten. Auf diese Weise wurde der Thorax geöffnet. Hierauf wurden zwei breite stumpfe Haken in die Wundöffnung eingesetzt und anseinander gezogen. Um die Iunge vor Verletzungen zu schützen, wurden die dort befindlichen Lungenlappen mit Gaze zugedeckt und dann unter leichtem Druck medialwärts verschoben. Dadurch wurde der sympathische Grenzstrang und Vagus gut sichtbar gemacht. Der Grenzstrang wurde bis zum Ganglion stellatum verfolgt; mit einer gebogenen Hakenpinzette fasste man 
den letzteren und legte ihn mit Hilfe einer gebogenen Pinzette frei. Die Rami communicates für die unteren Hals- sowie die oberen Brustnerven wurde mit einem von Cl. B ernard angegebenen Haken ${ }^{17}$ ) abgetrennt. Darauf wurde die Ansa subclavia nach oben bis zum unteren Halsganglion verfolgt und dicht unterhalb desselben herauspräpariert. Die von der Ansa sowie von dem Ganglion stellatum herabziehenden Äste wurden völlig freigelegt und möglichst weit cranialwärts durchschnitten. Der sich caudal von diesem Ganglion fortsetzenden Grenzstrang wurde nach unten hin bis unterhalb des achten Brustganglions durch Abtrennung von dessen Rami communicantes und Durchschneidung der vom ihm ausgehenden feinen Äste frei präpariert und dann in der Höhe der achten Rippe durchschnitten und entfernt.

Um den linken Vagusstamm gut präparieren zu können, haben wir zwei Fäden unter den Vagusstamm, oberhalb sowie unterbalb des Aortenbogens, geführt und ohne grosse Kraftanwendung lateralwärts gezogen. Die von diesem Stamme abzweigenden Äste wurden dann freigelegt und ein möglichst grosses Stück derselben unter sorgfältiger Blutstillung entfernt und damit natürlich auch der Recurrens sin. Auf diese Weise wurde der Vagus dicht unterhalb des Ganglion cerv.inf. caudalwärts bis zu seiner Abzweigungsstelle zum Oesophagus frei präpariert und der Stamm selbst dort zurückgelassen, damit dem Auftreten von Verdauungsstörungen vorgebeugt wurde.

Um die Äste, die aus dem Stamme des linken Vagus sowie aus dem linken unteren Halsganglion entspringen, um nach dem Herzen zu verlaufen, vollständig zu durchschneiden, ist es ferner immer bei Hunden nötig den Vagosympathicus am unteren Hals bis unterhalb des unteren Halsganglions freizulegen, um die daraus entspringenden Äste abzuschneiden.

Auf der rechten Seite wurde der obere thorakale Grenzstrang in der oben beschriebenen Weise vom Ganglion stellatum bis zum achten Brustganglion herausgenommen. Der Stamm des rechten Vagosympathicus wurde am Hals freigelegt und kurz oberhalb des unteren Halsganglions durchschnitten. Dabei wurde ein 2-3 cm langes Stück desselben entfernt.

Um den Einfluss des humoralen herzbeschleunigenden Agens aus der Leber auszuschalten, durchschnitten wir das hauptsächlich die Arteria hepatica umfassende Nervengeflecht in derselben Weise, wie es von den Bostoner Physiologen beschrieben wurde. Es wurde möglichst lang durchschnitten.

Diese Gesamtoperation wurde unter Einhaltung strenger Asepsis in drei bis vier Malen in Abständen von 1-3 Wochen ausgeführt.

An den Tieren, die diese Operationen gut überstanden hatten, wurden nun unsere Abkühlungsversuche angestellt. Die Minutenfrequenz des Herzschlages wurde durch sorgfältiges Auszählen während einer ganzen Minute mit Hilfe eines Stethoscopes und einer Stoppuhr erhalten. Zur Feststellung der Ruhezahl wurde jedes Tier, das etwa zwanzig Stunden lang keine Nahrung erhalten hatte (in unserem Laboratorium wurde den Tieren einmal am Tage Futter gegeben, diesen Hunden z. B. um 2 Uhr nachmittags), ungefesselt auf

17) Cl. Bernad, Leçons de physiologie expérimentale I, 1855 Paris, 337. 
einen Tisch im Laboratorium gebracht und solange in Ruhe gelassen, bis die Pulsfrequenz konstant geworden war. Während des ganzen Verlaufes des Versuches wurden gleichbleibende Versuchsbedingungen eingehalten. Die Beeinflussung der körperlichen und seelischen Ruhe der Tiere durch die Anwesenheit fremder Personen, Geräusche von aussen und andere störende Reize wurde nach Möglichkeit vermieden.

Um die Kälteapplikation im Ruhezustande der Tiere zu ermöglichen, wurde ein Gummirohr mit der vorher eingeführten Magenfistelkanüle verbunden und durch dieses eine bestimmte Menge kaltes Wasser mit einer Spritze in den Magen meistens in 1-3 Minuten eingeführt. Man musste gewöhnlich die Wiederherstellung der Ruhezahl abwarten, ehe man dieses letztere Verfahren, d.h. Einführung des kalten Wassers ausführt. Die Menge des eingeführten eiskalten Wassers $\left(0,5-1^{\circ} \mathrm{C}\right)$ betrug $300-550 \mathrm{ccm}$. Sie genügte um eine Wärmeschuld von aunähernd 2900-3100 kleinen Kalorien pro kg Körpergewicht herzustellen. Hierbei sei erwähnt, dass unsere Hunde eine grössere Wärmeschuld als die Cannonschen Katzen (etwa das Doppelte) aufwiesen.

\section{Versuchsergebnisse.}

Bei 8 Hunden, die die vollständige Denervierung desHerzens gut überstanden hatten, untersuchte ich 16 mal sowohl bei vorhandener wie bei ausgeschalteter Epinephrinsekretion den Einfluss der Abkühlung, die durch die Einführung kalten Wassers durch die Magenfistel in den Magen vorgenommen wurde, auf das Verhalten der Schlagfrequenz des Herzens. Dass die Einführung des kalten Wassers in den Magen durch eine Magenfistel für diesen Zweck sehr geeignet ist, ergibt sich aus der Tatsache, dass während der Darreichung des Wassers sich jedes Tier fast ganz ruhig verhielt und die Pulszahl nahezu unverändert blieb oder nur ganz wenig beschleunigt wurde. Bei keinem der die Ansschaltung der extrakardialen Herznerven überlebenden Tiere liess sich eine respiratorische Arrhythmie des Herzschlages nachweisen; diese war bei unseren Tieren in der Regel bald nach vollendeter Denervierung des Herzens verschwunden, d. h. nach Durchschneidung des rechten Vagosympathicus am Hals, womit die gesamte Operation abgeschlossen wurde. Die Bostoner Physiologen sehen die Abwesenheit der respiratorischen Arrhythmie als ein sicheres Anzeichen für die vollkommene Entnervung des Herzens an. Bei unseren Versuchen wurde die Schlagfrequenz nach Abschluss der Operation viel beständiger und durch Körper- oder Gemütsbewegung viel weniger beeinflussbar als vorher. Eine Stunde lang vor der Wassereinführung, währenddessen sich das Tier freiwillig sitzend oder liegend auf einem Tisch befand, behielt die Schalgfrequenz eine fast kon- 
stante Grösse mit nur kleinen Schwankungen bei, einerlei ob das Nebennierenmark entfernt oder in seiner Funktion durch die Splanchnikotomie ausgeschaltet worden war oder noch nicht.

In der folgenden Tabelle und in den Figuren sind nun die Ergebnisse der einzelnen Versuche zusammengestellt.

1. Der Einfluss der Einführung von kaltem Wasser in den Magen auf die Schlagzahl des entnervten Herzens bei Hunden mit erhaltenen Nebennieren.

In Übereinstimmung mit den Angaben von Cannon und seinen Mitarbeitern konnte ich in sämtlichen 10 Versuchen an 8 Hunden nach der Einführung eiskalten Wassers in ihren Magen eine beträchtliche Schlagfrequenzsteigerung des denervierten Herzens feststellen. Bei unseren Hunden, bei denen durch eiskaltes Wasser eine Wärmeschuld von annähernd 2900-3100 kleinen Kalorien pro kg Körpergewicht hergestellt wurde, begann die Pulsfrequenz schon in 3-7 Minuten nach dem Beginn der Wassereinführung, welche gewöhnlich etwa zwei Minuten in Anspruch nahm, zuzunehmen und erreichte ihr Maximum in etwa 15-25 Minuten. (In einigen Fällen ist man wohl nicht berechtigt den Zeitpunkt, bei dem die Beschleunigung zuerst auftrat, ziemlich genau anzugeben, weil die erste Bestimmung der Pulszahl nach der Wassereinführung nach Ablauf einiger Minuten vorgenommen wurde und schon dabei eine bedeutende Akzeleration zu beobachten war.) In einem Falle trat das Maximum nach etwa einer Stunde ein (Hund 35). Die Schlagfrequenz übertraf die Ausgangsfrequenz ad maximum um 19-41 Schläge pro Minute, d. h. um 12-39\%. Bei Hund 24 bewirkte die Abkühlung mit einer Wärmeschuld von 2400 kleinen Kalorien pro kg eine Frequenzvermehrung um 31 Schläge pro Minute, deren Maximum in 17 Minuten erreicht wurde. Meistens hielt die vermehrte Schlagzahl, etwa 15-20 Schläge pro Minute über dem Anfangswert, etwa fünfzehn bis dreissig Minuten an und stieg dann ab, um meistens 1-2 Stunden nach der Kälteapplikation ihre Ausgangszahl wieder zu erreichen. In einigen Fällen schien, wie bei Hund Nr. 35, die Wiederherstellung des Ausgangswertes noch etwas längere Zeit in Anspruch zu nehmen.

Bei ein und demselben Tiere waren die durch die gleiche Wärmeschuld hervorgerufenen Frequenzsteigerungen nicht immer in demselben Ausmasse vorhanden. In zwei Versuchen mit Hund 23, bei dem eine Wärmeschuld von 3100 kleinen Kalorien pro kg hergestellt worden war, vermehrte sich die Pulszahl maximal um 23 Schläge in 26 Minuten bzw. um 41 Schläge in 17 Minuten. Bei Hund 30 betrug die maximale Frequenzvermehrung in zwei Untersuchungen 


\section{Tabelle.}

Der Einfluss der Einführung kalten Wassers in den Magen auf die Pulsfrequenz von Hunden mit ausgeschalteten extrakardialen Herznerven vor und nach der Ausschaltung der Epinephrinsekretion.

\begin{tabular}{|c|c|c|c|c|c|c|c|c|c|c|c|}
\hline \multirow{3}{*}{$\begin{array}{l}\dot{\vec{Z}} \\
\vec{z} \\
\ddot{\Xi} \\
\ddot{\Xi}\end{array}$} & \multirow{3}{*}{ 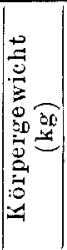 } & \multirow{3}{*}{ 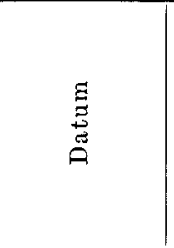 } & \multirow{3}{*}{ 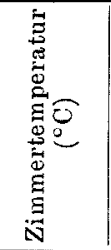 } & \multirow{3}{*}{ 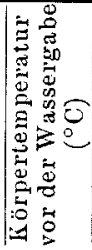 } & \multirow{3}{*}{ 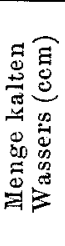 } & \multirow{3}{*}{ 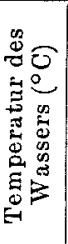 } & \multirow{3}{*}{ 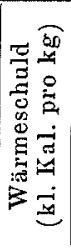 } & \multicolumn{3}{|c|}{ Herzschlag } & \multirow{3}{*}{ 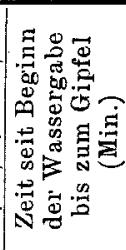 } \\
\hline & & & & & & & & \multirow{2}{*}{ 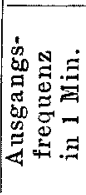 } & \multicolumn{2}{|c|}{$\begin{array}{c}\text { Maximale } \\
\text { Frequenz- } \\
\text { vermehrung }\end{array}$} & \\
\hline & & & & & & & & & in 1 & $\begin{array}{l}\text { in } \% \text { der } \\
\text { Ausgangs } \\
\text { frequenz }\end{array}$ & \\
\hline \multirow[t]{2}{*}{21} & 4,8 & 30. VII. 1932 & $31-31,5$ & 38,8 & 400 & 1 & 2900 & 114 & 32 & 28 & 16 \\
\hline & 4,0 & 5.VIIT. 1932 & $29.5-28,5$ & 38,5 & 330 & 0,5 & 3100 & 99 & 34 & 34 & 35 \\
\hline \multirow[t]{3}{*}{23} & 5,4 & 31. VIII. 1932 & 30 & 39,1 & 430 & 0,5 & 3100 & 114 & 23 & 20 & 26 \\
\hline & 5,8 & 5. IX. 1932 & 28 & 39,0 & 460 & 0,5 & 3100 & 108 & 41 & 39 & 17 \\
\hline & 5,5 & IX. 1932 & 19 & 39.0 & 450 & 0.5 & 3200 & 111 & 32 & 29 & 23 \\
\hline \multirow[t]{2}{*}{24} & 4,7 & IX. 1932 & $21-22$ & 38,2 & 300 & 1 & 2400 & 122 & 31 & 25 & 17 \\
\hline & 4,4 & IX. 1932 & $19-19,5$ & 38,9 & 300 & 1 & 2600 & 112 & 29 & 26 & 30 \\
\hline \multirow[t]{3}{*}{30} & 6,1 & 27. XII. 1932 & $18,5-20$ & 39,2 & 500 & 1 & 3100 & 155 & 19 & 12 & 24 \\
\hline & 6,8 & 1. 1933 & $17-16$ & 39,5 & 550 & 1 & 3100 & 128 & 23 & 18 & 21 \\
\hline & 6,5 & I. 1933 & $20,5-22,5$ & 38.9 & 550 & 1 & 3200 & 109 & 25 & 23 & 20 \\
\hline \multirow[t]{2}{*}{31} & 6,3 & II. 1933 & $18-21$ & 40,2 & 500 & 1 & 3100 & 116 & 25 & 22 & 19 \\
\hline & 60 & II. 1933 & $16,5-17,5$ & 39,8 & 480 & 1 & 3100 & 122 & 29 & 24 & 61 \\
\hline \multirow[t]{2}{*}{34} & 4,2 & II. 1933 & $19-22$ & 39.3 & 350 & 1 & 3100 & 132 & 33 & 25 & 13 \\
\hline & 40 & II. 1933 & $21-18,5$ & 39,1 & 350 & 1 & 3100 & 113 & 30 & 27 & 19 \\
\hline 35 & 4,6 & III. 1933 & $20, \overline{5}-18,5$ & 40,0 & 370 & 1 & 3100 & 129 & 28 & 22 & 59 \\
\hline 36 & 5,7 & 1. IV. 1933 & $17-16$ & 39,8 & 430 & 1 & 2900 & 143 & 25 & 18 & 23 \\
\hline
\end{tabular}

Hund Nr. 21. \& : 27. VI. 1932. 5,0 kg. 9:55-11:15 a.m. Das linke Ganglion stellatum mit dem ihm folgenden thorakalen Grenzstrange bis zum 8 . Brustganglion herausgenommen. Alle vom linken Vagus, ron dicht unterhalb des Ganglion cerv.inf. bis zum Oesophagus, abzweigenden Äste durchschnitten. $\quad 4$. VII. $1932.4,8 \mathrm{~kg} .9: 40-$ 10:43 a.m. Die Lebernerven durchschnitten. Die Magenfistel hergestellt. Stamm des linken Vagosympathicus am Hals freigelegt und alle aus diesem abgehenden Äste, bis unterhalb des Ganglion cerv. inf., durchschnitten. 15. VII. 1932 . 4,8 kg. 10:08-11:08 a.m. Das rechte Ganglion stellatum mit dem ihm folgenden thorakalen Grenzstrange bis zum 8. Brustganglion entfernt. 23. VII. 1932. 4,6 kg. 11:08-11:20 a.m. Der Stamm des rechten Vagosympathicus am Hals freigelegt und durchschnitten. 1. VIII. 1932. 4,5 kg. 10:10-11:18 a.m. Das Nebennierenmark auf beiden Seiten entfernt.

Hund Nr. 23. $q$ : 25. VII. 1932 . 5,0 kg. 9:55-10:45 a.m. Das linke Ganglion stellatum mit dem ihm folgenden thorakalen Grenzstrange freipräpariert und entfernt. Alle vom linken Vagus, von dicht unterhalb des Ganglion cerv, inf. bis zum Oesophagus abzweigenden Äste durchschnitten. 4. VIII. 1932 . 4,3 kg. 9:46-10:48 a.m. Das rechte Ganglion stellatum mit dem ihm folgenden thorakalen Grenzstrange bis zum 8. Brustganglion herausgenommen. 20. VII. 1932. 4,8 kg. 10:10-11:15 a.m. Der Stamm des linken Vagosympathicus am Hals freigelegt und alle von diesem abgehenden $\mathbf{Z}$ weige, bis unterhalb des Ganglion cerv. inf,, durchschnitten. Die Lebernerven durchschnitten. Die MIagenfistel hergestellt. 27. VIII. 1932. 5,5 kg. 9:55-10:08 a.m. Der rechte Vagosympathicusstamm am Hals freigelegt und durchschnitten. 15. IX. 1932. 6,0 kg. 9:38-10:45 a.m. Das Nebennierenmark auf beiden Seiten entfernt.

Hund Nr. 24. ㅇ: 20. VII. 1932. 4,2 kg. 9:45-11:10 a.m. Das linke Ganglion stellatum mit dem ihm folgenden thorakalen Grenzstrange bis zum 8. Brustganglion herausgenommen. Alle vom linken Vagus, von dicht unterhalb des Ganglion cerv. inf. bis zum Oesopha rus, a bgehenden Äste durchschnitten. 25. VIII. 1932 . 4,2 kg. 10:0-11:55 a.m. Der Stamm des linken Vagosympathicus am Hals freigelegt und alle von diesem abzweigenden $\ddot{\text { sste, }}$ bis unterhalb des Ganglion cerv, inf., durchschnitten. Die Lebernerven durchschnitten. Die Magenfistel hergestellt. 2. IX. 1932. 4,2 kg. 10:10-11:15 a.m. Das rechte Ganglion stellatum mit dem ihm folgenden thorakalen Grenzstrange 
bis zum 8. Brustganglion entfernt. 9. IX. 1932. 4,6 kg. 9:15-9:38 a.m. Der rechte Vagosympathiousstamm am Hals freigelegt und durchschnitten. $20 . \mathrm{IX} .1932 .4,6 \mathrm{~kg}$. 10:50 a.m.-12:15 p.m. Die beiderseitige Entfernung des Nebennierenmarkes volgenommen.

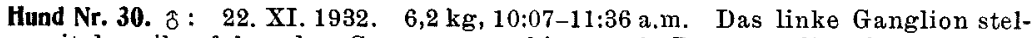
latum mit dem ihm folgenden Grenzstrange bis zum 8. Brustganglion freipräpariert und entfernt. Alle vom linken Vagus, von dicht unterhalb des Ganglion cerv. inf. bis zum Oesophagus, abzweigenden Äste durchschnitten. 29. XI. 1932 . 6,1 kg. 10:2511:23 a.m. Der linke Vagosympathicusstamm am Hals freigelegt und alle von diesem abziehenden $Z$ weige bis weit unterhalb des Ganglion cerv. inf, durchschnitten. Die Lebernerven durchschnitten. Die Magenfistel hergestellt. 6. XII, 1932. 6,5 kg. 10:3011:45 a.m. Das rechte Ganglion stellatum mit dem ihm folgenden Grenzstrange bis zum 8. Brustganglion freipräpariert und herausgenommen. 26. XII. $1932.6,1 \mathrm{~kg}$. 10:30-10:40a.m. Der Stamm des rechten Vagosympathicus an Hals freigelegt und durchschnitten. 7. I. 1933. 6,7 kg. 10:25-11:35 a.m. Das Nebennierenmark auf beiden Seiten entfernt.

Hund Nr. 31 \%: 24. XI. 1932. 5,7 kg. 10:20-11:34 a.m. Das linke Ganglion stellatum mit dem ihm folgenden thorakalen Grenzstrange bis zum 8 . Brustganglion freipräpariert und entfernt. Alle vom linken Vagus, von dicht unterhalb des Ganglion

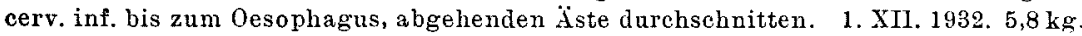
10:10-11:50 a.m. Der linke Vagosympathicussta $\mathrm{mm}$ am Hals freigelegt und alle vou diesem abzweigenden $\ddot{A} s t e$, bis weit unterhalb des Ganglion cerv. inf., durchschnitten. Die Lebernerven zerstört. Die Magenfistel hergestellt. 15. XIII. 1932. 6,4 kg. 10:2711:50 a.m. Das rechte Ganglion stellatum mit dem ihm folgenden thorakalen Grenzstrange bis zum 8 . Brustganglion herausgenommen. 30. I. 1933. 6,9 kg. 11:10-11:28 a.m. Der Stamm des rechten Vagosympathicus am Hals durchschnitten. 4. II. 1933. 6,3 kg. 4:05-4:58 p.m. Das Nebennierenmark beiderseitig entfernt.

Hund Nr. 34. $\delta:$ 26. I. 1933 . $5.3 \mathrm{~kg}$. 10:23-11:48 a.m. Das linke Ganglion stellatum mit dem ihm folgenden thorakalen Grenzstrange bis zum 8 . Brustganglion freipräpariert und entfernt. Alle rom linken Vagus, von dicht unterhalb des Ganglion cerv. inf. bis zum Oesophagus, abgehenden Äste durchschnitten. 3. II. $1933.4,9 \mathrm{~kg}$. 10:15-11:33 a.m. Der linker Vagosympathicusstamm am Hals freigelegt und alle von diesem abzweigenden Äste, bis weit unterhalb des Ganglion cerv. inf, durchschnitten. Die Lebernerven durchschnitten. Die Magenfistel hergestellt. 16. II. $1933.4,8 \mathrm{~kg}$. 10:28-11:33 a.m. Das rechte Ganglion stellatum mit dem ihm folgenden Grenzstrange bis zum 8. Brustganglion herausgenommen. 20. II. 1933. 4,7 kg. 11:02-11:20 a.m. Der rechte Vagosympathicusstamm am Hals durchschnitten. 24. II. 1933 . 4,2 kg. 9:2410:30 a.m. Nervi splanchnici auf beiden Seiten durchschnitten.

Hund Nr. 35. 우 : 27. I. 1933. 4,7 kg. 10:27-11:55 a.m. Das linke Ganglion stellatum mit dem ihm folgenden thorakalen Grenzstrange bis zum 8 . Brustganglion freipräpariert und herausgenommen. Alle vom linken Vagus, von dicht unterhalb des Ganglion cerv. inf. bis zum Oesophagus, abzweigenden Äste durchschnitten. 3. II. 1933. 4,5 kg. 11:03 a.m.-12:28 p.m. Der linke Vagosym pathicusstamm am Hals freigelegt und alle von diesem abgehenden $Z$ weige, bis weit unterhalb des Ganglion cerv. inf., durchsohnitten. Die Lebernerven ausgeschaltet. Die Magenfistel hergestellt. 10. II. 1933. $4,5 \mathrm{~kg} .10: 36 \mathrm{a} . \mathrm{m} .-12: 02$ p.m. Das rechte Ganglion stellatum mit dem ihm folgen. den thorakalen Grenzstrange bis zum 8. Brustganglion herausgenommen. 1. III. 1933. 5,0 kg. 11:02-11:15 a.m. Der Stamm des rechten Vagosympathicus am Hals durchschnitten.

Hund Nr. 36. $\&$ : $\quad$ 9. II. 1933. 5,3 kg. 10:28 a.m.-12:08 p.m. Das linke Ganglion stellatum mit dem ihm folgenden thorakalen sympathischen Grenzstrange bis zum 8 . Brustganglion freipräpariert und herausgenommen. Alle vom linken Vagusstamme, von dicht unterhalb des Ganglion cerv. inf. bis zum Oesophagus, abzweigenden Äste durchschnitten. 17. II. 1933 . $5,2 \mathrm{~kg}$. 10:20-10:55 a.m. Der linke Vagosympathicusstamm am Hals freigelet und alle von ihm abzweigenden $\ddot{A}$ ste, bis weit unterhalb des Ganglion cerv. inf., durchschnitten. 25. II, 1933. 5,2 kg. 1:30-3:07 p.m. Die Lebernerven durchschnitten. Die Magenfistel hergestellt. 2. III. 1933. 5,3 kg. 10:28-11:30 a.m. Das rechte Ganglion stellatum mit dem ihm folgenden thorakalen Grenzstrange bis zum 8. Brustganglion herausgenommen. 31. III. 1933. 6,0 kg. 11:50 a.m,-12:01 p.m. Der Stamm des rechten Vagosympathicus am Hals durchschnitten. 4. IV. 1933. $5,2 \mathrm{~kg}$. 2:30-3:05 p.m. Nervi splanchnici auf beiden Seiten durchschnitten. 
Abb. 2 .
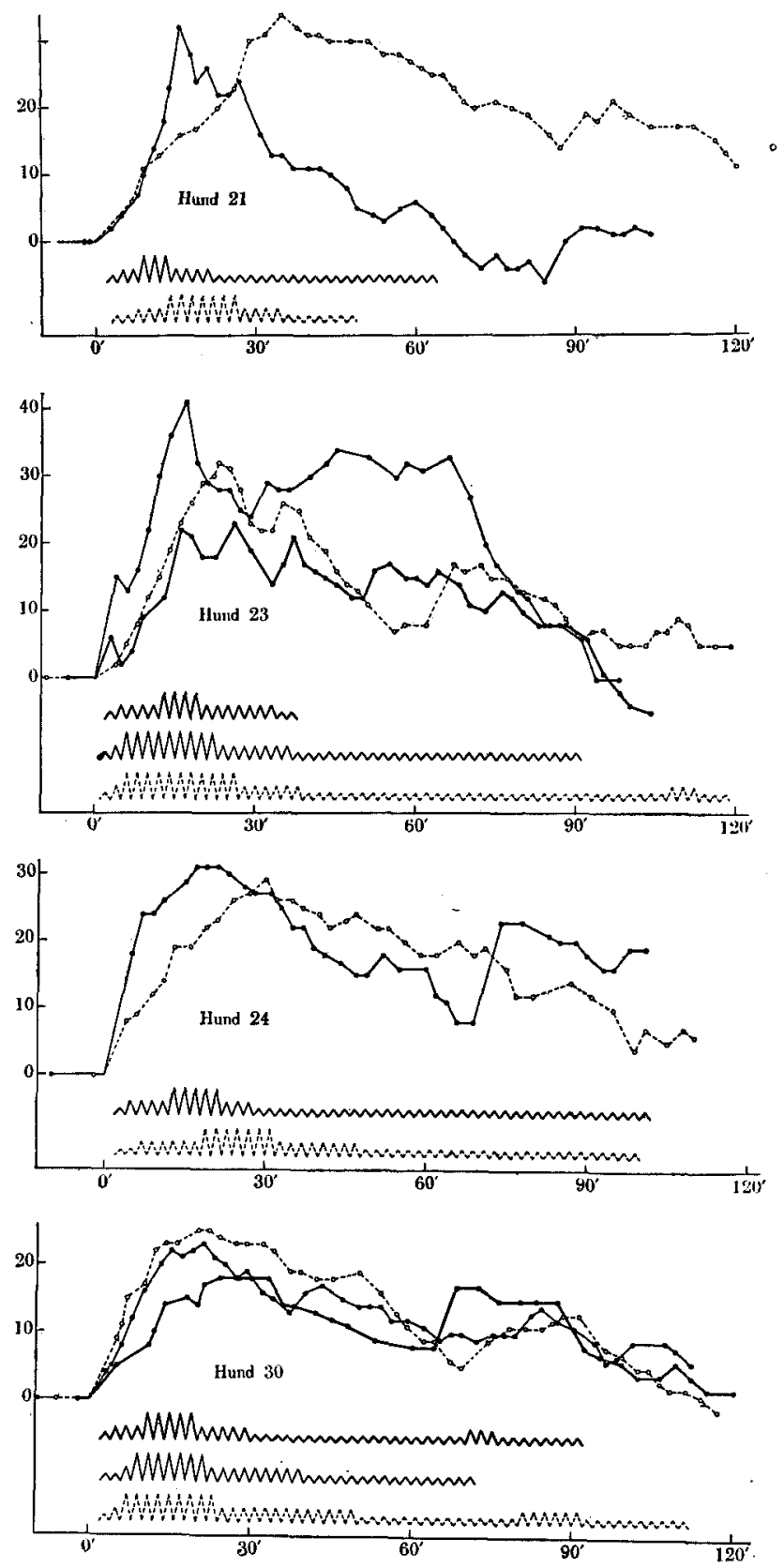
Abb. 2. (Fortsetzung)
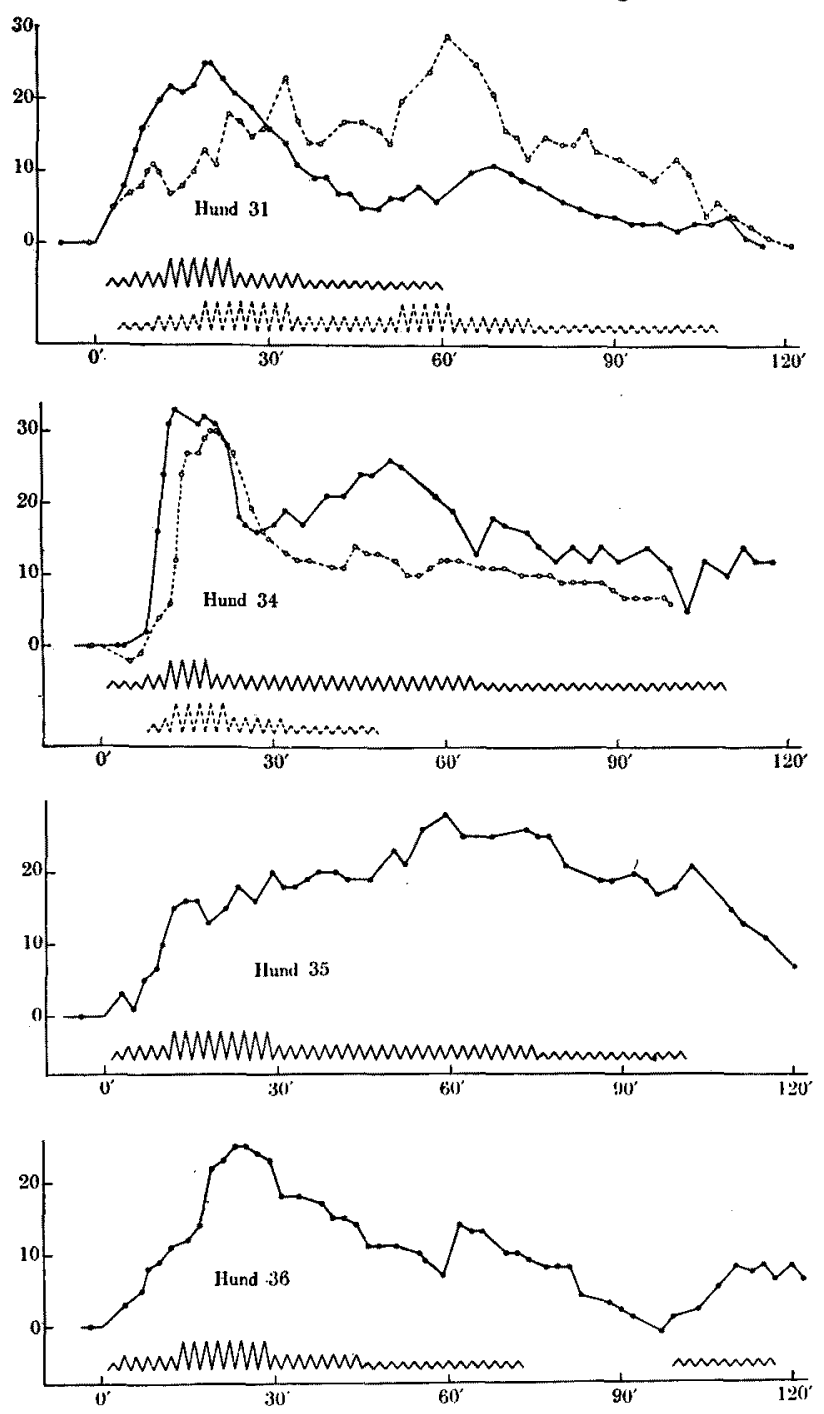

Pulsfrequenzsteigerung des entnervten Herzens nach der Einführung von kaltem Wasser in den Magen. Abszisse: Zeit in Minuten. Ordinate: Pulsfrequenzsteigerung pro Minute. Die ausgezogenen Linien stellen die Daten bei intakten Nebennieren und die gestrichelten Linien diejenigen nach der Ausschaltung der Epinephrinsekretion dar. Die Zickzacklinien das Zittern. Die Höhe derselben entspricht der Stärke des Zitterns: die höchste das kontinuierliche Zittern nnd die anderen das Zittern während der Inspiration. (Bei Hund 23 und 30 ist die Frequenzvermehrung sowie das Zitteru beim ersten und zweiten Experiment vor der Demedullation mit dicken und dünnen Linien dargestellt.) 
mit der Wärmeschuld von 3100 kleinen Kalorien pro kg 19 und 23 Schläge pro Minute; diese Beschleunigung wurde in 24 bzw. 21 Minuten erreicht. Ebenso treten anch Verschiedenheiten in der Frequenzsteigerung bei verschiedenen Individuen auf: bei einer Wärmeschuld von $3100 \mathrm{kleinen} \mathrm{Kalorien} \mathrm{pro} \mathrm{kg} \mathrm{stieg}$ die Pulsfrequenz bei Hund 31 maximal um 25 Schläge in 19 Minuten und bei Hund $35 \mathrm{um} 28$ Schläge in einer Stunde. Bei Hund 21 wurde die maximale Vermehrung von 32 Schlägen in 16 Minuten bei einer Wärmeschuld von 2900 kleinen Kalorien pro kg erhalten, während bei Hund 36 die bei der gleichen Wärmeschuld festgestellte maximal Vermehrung 25 Schläge betrug und in 23 Minuten erreicht wurde.

Oben ist nur die maximale Beschleunigung in Betracht gezogen, aber diese Gegenüberstellung zeigt die Sachlage nicht besonders deutlich. Ein Vergleich der Kurven in Abb 2 zeigt das hier besprochene Verhältnis viel einleuchtender.

Aus diesen Versuchen ergibt sich also, dass die Frequenzsteigerung des entnervten Hundeherzens unter dem Kälteeinflusse mit einer Wärmeschuld von etwa 3000 kleinen Kalorien pro kg etwa $20-30 \%$ des Anfangswertes beträgt, annähernd ähnlich wie bei den Versuchen der Bostoner Physiologen, bei denen die Schlagfrequenz des denervierten Katzenherzens nach der Ablühlung durch eine Wärmeschuld von 1500-2000 kleinen Kalorien pro kg um 23-28 Schläge gesteigert wurde.

Die Kälteapplikation liess bei unseren Tieren in der Regel ein merkliches Schaudern gleichzeitig mit der Frequenzsteigerung des Herzens zustande kommen.

So trat in sämtlichen Versuchen 2-3Minuten nach dem Anfang der Kälteapplikation ein leichtes Muskelzittern während der Inspiration auf ; es wurde immer beträchtlicher, bis nach 5-12 Minuten ein kontinuierliches Zittern, unabhängig von der Atmung, zu beobachten war. Die Dauer des letzteren betrug etwa 7-20 Minuten, in der Mehrzahl der Fälle 10 Minuten, während das Maximum der Schlagfrequenzsteigerung entweder gleichzeitig oder etwas später erreichtwurde. Nach dieser Periode blieb das Zittern wieder während der Inspiration bestehen und wurde dann allmählich schwächer. Das Zittern hörte 40 Minuten bis etwa zwei Stunden nach der Wassereinführung auf, während die Wiederherstellung der Schlagzahl manchmal noch später eintrat. Als in seltenen Fällen im späteren Stadium wieder ein starkes Zittern auftrat, wurde auch die Pulszahl fast gleichzeitig wieder vermehrt gefunden. Doch gab es noch andere Fälle, in denen ein Wiederauftreten der Pulsbeschleunigung nicht von Zittern oder einer $\mathrm{Zu}$ nahme des Zitterns begleitet war. 
In den an Katzen angestellten Versuchen fanden die Bostoner Physiologen ebeufalls, dass zur Zeit wo die Pulsfrequenz gewöhnlich maximal gesteigert war, auch das Zittern meistens am stärksten auftrat.

Bei gleicher Wärmeschuld war der Ablauf des Zitterns nicht immer gleich. Bei Hund 23 bewirkte die Abkühlung mit einer Wärmeschuld von 3100 kleinen Kalorien pro kg im ersten Versuch ein kontinuierliches Zittern von 10 Minuten Dauer 12 Minuten nach der Wasserdarreichung, während im zweiten Versuch mit gleicher Wärmeschuld ein solches von 15 Minuten Dauer nach 5 Minuten in Erscheinung trat; in diesen beiden Versuchen schwand das Zittern 40 bzw. 90 Minuten nach der Wasserdarreichung. In zwei Versuchen bei Hund 30, mit einer Wärmeschuld von 3100 kleinen Kalorien pro kg war das Zittern 10 Minuten lang kontinuierlich und dauerte $10 \mathrm{bzw}$. 15 Minuten an; das Zittern hörte nach 90 bzw. 70 Minuten nach der Kälteapplikation auf.

Dass diese Schlagfrequenzvermehrung des entnervten Herzens eine Reaktion auf die Kältewirkung des Wasser ist, ergibt sich aus der Tatsache, dass nach der Einführung einer ebenso grossen Menge körperwarmen Wassers in den Magen die Schlagfrequenz fast unbeeinflusst bleibt. Das am 31. VII. an Hund 21 gewonnene Resultat stellt ein Beispiel hierfür dar. Bei diesem Hunde fanden wir nach Darreichung von $400 \mathrm{ccm}$ Wasser von annähernd $38^{\circ}, 5 \mathrm{C}$ eine Schlagfrequenzsteigerung um 7 Schläge in 2 Minuten, um 4 Schläge in 7 Minuten und um 2 Schläge in 9 Minuten statt einer Beschleunigung um 32 Schläge in 16 Minuten nach Applikation von $400 \mathrm{ccm}$ Wasser von $1^{\circ}$. Dabei trat das Zittern natürlich nicht auf. Eine Durchsicht der von den oben erwähnten Forschern mitgeteilten Tabelle ${ }^{18)}$ zeigt, dass die Zufuhr von $162 \mathrm{bzw} .146 \mathrm{ccm}$ Wasser von $33^{\circ}$ und $31^{\circ}$ eine Frequenzvermehrung verursachte, die in beiden Fällen unmittelbar danach 36 Schläge pro Minute betrug und etwa 10-15 Minuten anhielt. Wenn man ausser Aclht lässt, dass Katze 26 sich bei einem ähnlichen Versuche sehr aufregte, was eine riesige Pulsbeschleunigung zur Folge batte, so ist diese Frequenzvermehrung eine viel grössere, als die bei unserem Tieje beobachtete, was wohl wahrscheinlich eine Folge der durch das wenig. geeignete Verfahren bewirkten Störung des Ruhezustandes der Tiere ist. Die Temperatur des Wassers war auch etwas niedriger als die Körpertemperatur der Katze.

Die Kälteapplikation verursachte unter diesen Bedingungen eine mehr oder weniger deutliche aber nicht hoch gradige Erniedrigung der Körpertemperatur. Bei Hund 23, bei dem eine Wärmeschuld von 3100 kleinen Kalorien pro kg durch Wassereinführung hergestellt worden war, sank die Analtemperatur in einem Versuche am 31. VIII. um

18) Cannon, Querido, Britton und Bright, (1), S. 476. 
$0,5^{\circ}$ (von $39^{\circ}, 1$ auf $\left.38^{\circ}, 6\right) 20$ Minuten nach der Kälteapplikation. In dem am 5. IX. ausgeführten Versuche wurde eine Temperaturerniedrigung von $0^{\circ}, 9 \mathrm{C}$ (von $39^{\circ}, 0$ auf $38^{\circ}, 1$ ) nach 15 Minuten nachgewiesen. In zwei Versuchen an Hund 30 war die in 25 bzw. 30 Minuten bestimmte Temperaturerniedrigung $1^{\circ} \mathrm{C}$, und zwar sank die Temperatur von $39^{\circ}, 2$ auf $38^{\circ}, 1$ bzw. $39^{\circ}, 5$ auf $38^{\circ}, 4$. Der gleiche Temperaturabfall wurde bei Hund 34 und 35 in 20 bzw. 15 Minuten gefunden; die Temperatur sank von $39^{\circ}, 3$ auf $38^{\circ}, 2 \mathrm{bzw}$. von $40^{\circ}, 0$ auf $38^{\circ}, 7$. Bei Huud 21 und 36 , mit einer Wärmeschuld von 2900 kleinen Kalorien pro kg sank die Temperatur um $0^{\circ}, 9 \mathrm{C}$ (von $38^{\circ}, 8$ auf $37^{\circ}, 9$ ) bzw. $1^{\circ}, 2$ (von $39^{\circ}, 8$ auf $38^{\circ}, 6$ ) in 15 Minuten ab. Bei Hund 24 mit einer Wärmeschuld von 2400 kleinen Kalorien lag die 15 Minuten nach der Kälteapplikation gemessene Temperatur um $0^{\circ}, 6$ unter. dem Anfangswert von $38^{\circ}, 2$.

Bei einem Kälteeinfluss von genannter Grösse war die Anfangstemperatur meistens in 80-120 Minuten nach der Kälteapplikation erreicht.

2. Einfluss der Einführung von kaltem Wasser in den Magen auf die Schlagfrequenz des entnervten Hundeherzens nach der Ausschaltung der Epinephrinsekretion.

Um zu prüfen, ob die unter dem Kälteeinflusse feststellbare Schlagfrequenzvermehrung des denervierten Hundeherzens eine Folge der Epinephrinmehrabgabe ist, empfahl es sich, bei ein und demselben Tiere nach Entfernung des Nebennierenmarkes die Abkühlungsversuche noch einmal vorzunehmen. Es sei hier die Darstellung der diesbezüglichen Resultate gegeben.

Bei 5 Hunden (Hund 21, 23, 24, 30 und 31), bei denen die Frequenzsteigerung des Herzschlages bei einer gewissen Wärmeschuld festgestellt worden war, wurde die Markentfernung der Nebennieren vorgenommen. Diese Operation wurde durch einen einzigen Eingriff vom Rücken aus in üblicher Weise durchgeführt. Bei einem Hunde (Nr. 34) wurden dieNn. splanchnici doppelseitig vom Rücken aus blossgelegt und in einer Länge von $1 \mathrm{~cm}$ herausgeschnitten.

Das vollständige Gelingen der Markentfernung wurde nach dem Tode der Tiere, der nach Beendigung der anderen Experimente, wie Laufen, afferente Reizung etc., erfolgte, freundlicherweise von Herrn Professor Nas u vom Pathologischen Institut, makroskopisch sowie mikroskopisch, ausgeführt, und zwar war bei den hier zitierten Hunden der Operationserfolg tadellos.

Wie aus der Tabelle und den Abbildungen ersichtlich ist, war die 
Schlagfrequenzsteigerung bei der Kälteapplikation auch nach der Ausschaltung des Epinephrineinflusses sehr deutlich vorhanden.

Bei Hund $21 \mathrm{kam}$ durch eine Wärmeschuld von 3100 kleinen Kalorien pro kg nach Entfernung des Nebennierenmarkes die maximale Herzbeschleunigung von 34 Schlägen pro Minute, nämlich $34 \%, 35$ Minuten nach der Wassereinführung zustande, während bei intakten Nebennieren eine solche von 32 Schlägen, nämlich $28 \% 16$ Minuten nach der Kälteapplikation mit einer Wärmeschuld von 2900 kleinen Kalorien pro kg hervorgerufen wurde. Dieselben Resultate wurden bei vier anderen Hunden beobachtet.

Bei diesem Hunde wurden $330 \mathrm{ccm}$ kaltes Wasser $\left(0,5^{\circ} \mathrm{C}\right)$, die einer Wärmeschuld von 3000 kleinen Kalorien pro kg entsprechen, am Tage nach der Markoperation wie üblich in den Magen eingeführt. Dann kam nicht nur keine Beschleunigung des Pulses zur Tage, sondern das Herz schlug sogar eher langsamer, so dass eine Verminderung von 8 Schlägen pro Minnte, die maximale Verminderung, 20 Minuten nach dem Anfang der Wassereinführung notiert wurde. Eine etwas verlangsamte Frequenz und schwaches Zittern dauerten etwa 90 Minuten an. Dieser negative Ausfall entspricht den Angaben Cannons über den Einfluss der Anästhesie sowie operativer Eingriffe auf das entnervte Herz. Nach diesem Befunde stellten wir niemals wieder am Tage nach der Markoperation einen eigentlichen Versuch, wie Abkühlung, Fesselnng etc. an. Wir machten dagegen einige Tage nach der Markoperation unseren Versuch und waren imstande eine Pulsbeschleunigung durch Abkühlung, die an Intensität derselben vor der Demedullation gar nicht nachstand, zu beobachten. Dieses Resultat beweist, dass nur eine kurze Frist von einigen Tagen genügt, um den Einfluss der Anästhesie und dergleichen mehr auf die Reaktion des entnervten Herzens vollständig auszuschalten.

Bei Hund 23, bei dem in 2 Versuchen vor der Markentfernung die maximale Frequenzsteigerung bei einer Wärmeschuld von 3100 kleinen Kalorien pro kg 23 bzw. 41 Schläge, nämlich $20 \%$ bzw. $39 \%$ des Anfangswertes betragen hatte, wurde nach der Operation die Pulszahl bei einer Wärmeschuld von 3200 kleinen Kalorien pro $\mathrm{kg}$ um 32 Schläge, nämlich $29 \%$ in 23 Minuten gefördert.

Bei Hund 24 betrug bei entferntem Nebennierenmark die durch eine Wärmeschuld von 2600 kleinen Kalorien pro $\mathrm{kg}$ bedingte Herzbeschleunigung 29 Schläge, nämlich $26 \%$ und wurde in 30 Minuten erreicht; bei unversehrten Nebennieren machte sie bei einer Wärmeschuld von 2400 kleinen Kalorien pro $\mathrm{kg} 31$ Schläge, nämlich $25 \%$ aus und wurde in 17 Minuten beobachtet.

In zwei Versuchen an Hund 30 führte die Abkühlung mit einer Wärmeschuld von 3100 kleinen Kalorien pro kg bei intaktem Nebennierenmark eine Herzbeschleunigung von 19 bzw. 23 Schlägen (12 
bzw. 18\%) in 24 bzw. 21 Minuten herbei, nach der Markentfernung. ergab sich mit einer Wärmeschuld von 3200 kleinen Kalorien pro kg eine Beschleunigung von 25 Schlägen (23\%) in 20 Minuten.

Bei Hund 31 betrug die maximale Frequenzvermehrung infolge der Abkühlung mit einer Wärmeschuld von 3100 kleinen Kalorien pro $\mathrm{kg}$ vor und nach der Entfernung des Nebennierenmarkes 25 bzw. 29 Schläge (22 bzw. 24\% der Ausgangszahl); sie wurde in 19 bzw. 61 Minuten erreicht.

Die Abbildungen auf S. 560 u.561, worauf die Daten vor der-Demedullation mit ausgezogenen Linien und diejenigen nach der Operation gestrichelten angegeben sind, zeigen noch deutlicher, dass die Pulsbeschleunigungen durch Einführung von kaltem Wasser in den Magen vor und nach der Demedullation abgesehen von geringen Schwankungen in jedem Experimente von derselben Grösse und Dauer waren und die gleiche Steilheit zeigten, mit der die Beschleunigung ihren Gipfel erreichte. So war sie z. B. bei einem Hunde vor der Demedullation von grösserem Umfange als hinterher, aber bei einem anderen verhielt sie sich gerade umgekehrt.

Dass ein Parallelismus zwischen dem Verlauf des Zitterns und dem Verlauf der Herzbeschleunigung vorliegt, konnte ich nach der Ausschaltung der Epinephrinsekretion nachweisen. Auch nach der Entfernung des Nebennierenmarkes trat das Zittern bei der Inspiration 2-4 Minuten nach dem Beginn der Kälteapplikation auf und wurde allmählich stärker, bis es endlich nach etwa 5-20 Minuten in ein kontinuierliches übergegangen war. Das letztere dauerte etwa 10-20 Minuten lang an, währenddessen die Schlagfrequenz, abgesehen von Hund 21, in der Regel ihr Maximum erreichte.

Bei den Hunden 23 und 30 entwickelte sich das kontinuierliche Zittern in 5 Minuten nach der Kälteapplikation und dauerte etwa 20 Minuten lang an, das Maximum der Frequenzvermehrung des Herzschlages trat wie oben erwähnt, in etwa 20 Minuten ein. Bei diesen Tieren schwand das Zittern gleichzeitig mit der fast vollständigen Wiederherstellung der Ausgangsfrequenz 120 Minuten nach der Wassergabe. Bei Hund 30 wurde das Zittern etwa 80 Minuten nach der Kälteapplikation verstärkt, während sich gleichzeitig auch die Pulszahl vermehrte; beide Vorgänge dauerten etwa 10 Minuten.

Bei Hund 21, 24 und 31, bei denen das kontinuierlicheZittern nach 15, 20 bzw. 20 Minuten sichtbar wurde und etwa 15 Minuten lang anhielt, trat die maximale Schlagfrequenzvermehrung in $35,30 \mathrm{bzw}$. 60 Minuten nach der Wassereinführung in den Magen ein. Die Puls- 
beschleunigung trat also bei Hund 21 viel später auf, und zwar erst dann als das kontinuierlicheZittern dem intermittierenden Platz machte. Bei diesem Hunde verschwand das Zittern 50 Minuten nach der Wassergabe, wobei die Schlagfrequenz mit etwa 10 Schlägen über der Ruhezahl lag. Bei Hund $31 \mathrm{kam}$ von neuem eine grössere Beschleunigung von 27 Schlägen pro Minute etwa 60 Minuten nach der. Wassereinführung zustande, gleichzeitig zitterte der Hund heftig und kontinuierlich. Bei Hund 24 und 31 verschwand das Zittern etwa 100 Minuten nach der Wassergabe, während die Frequenzsteigerung noch länger anbielt, nämlich etwa 120 Minuten lang oder auch mehr.

Diese Experimente haben einen Aufschluss über einen etwaigen Zusammenhang zwischen der vermehrten Epinephrinsekretion und dem Auftreten des Zitterns im Sinne Cannons ${ }^{19}$ nicht erbracht. Nach der Demedullation danerte das Zittern bei Hund 23, 30 und 31 länger, aber das Gegenteil war bei Hund 21 und 34 (Splanchnikotomie) der Fall, während es beiHund 24 vor und nach der.Demedullation fast gleich lange anhielt. In jedem Fall sank die Körpertemperatur nach der Einführung des kalten Wassers anschliessend um etwa 1-2 Grade. Bei Hund 21 sank die Temperatur von $38^{\circ}, 5$ auf $38^{\circ}, 1$ in 20 Minuten, auf $37^{\circ}, 8$ in 35 Minuten und auf $36^{\circ}, 3$ in 75 Minuten; sie stieg wieder auf $38^{\circ}, 4$ in 110 Minuten und auf $38^{\circ}, 5$ in 130 Minuten. Bei Hund 23 sank die Temperatur von $39^{\circ}, 0$, auf $37^{\circ}, 9$ nämlich um $1^{\circ}, 18$ Minuten nach der Einführung des Wassers und stieg wieder auf $38^{\circ}, 0$ in $30 \mathrm{Mi}-$ nuten auf $38^{\circ}, 3$ in 45 Minuten und auf $38^{\circ}, 9$ in 2 Stunden. Bei Hund 24, bei dem die Temperaturmessung während der ersten halben Stunde nach der Kälteapplikation leider nicht ausgeführt wurde, war dieTemperatur anfangs $38^{\circ}, 9,35$ Minuten nach der Kälteapplikation $38^{\circ}, 3$ nach 80 Minuten $38^{\circ}, 9$ und nach 120 Minuten $39^{\circ}, 0$. Bei Hund 30 sank sie von $38^{\circ}, 9$ auf $38^{\circ}, 1$, nämlich um $0^{\circ}, 8$ in 15 Minuten; die 50,95 und 125 Minuten nach der Kälteapplikation bestimmten Temperaturen waren $38^{\circ}, 3,38^{\circ}, 6 \mathrm{bzw} .38^{\circ}, 9$. Hund 31 zeigte sich eine Temperaturerniedrigung von $39^{\circ}, 8$ auf $37^{\circ}, 7$, also um $2^{\circ}, 1$ in 20 Minuten; die Temperatur stieg auf $37^{\circ}, 9$ in 40 Minuten, auf $39^{\circ}, 2$ in 90 Minuten, und auf $39^{\circ}, 5$ in 125 Minuten.

Ferner war der Körpertemperaturabfall bei Hund 21 und 24 vor und nach der Demedullation beinahe gleich, bei Hund 30 nach der Operation etwas kleiner und bei den übrigen beiden Hunden etwas grösser.

19) Cannon, Querido, Britton und Bright, (1), 486. f. 
Im Zusammenhang mit den oben beschriebenen Versuchsresultaten kommen wir nun noch auf einen Versuch an Hund $34 \mathrm{zu}$ sprechen, bei dem der Einfluss der beiderseitigen Durchtrennung der Nn. splanchnici auf die Beschleunigung des entnervten Herzens bei der Abkühlung untersucht wurde. In einem 3 Tage vor der Splanchnikotomie ausgeführten Versuch reagierte dieser Hund, wie oben erwähnt, auf die Einführung von $350 \mathrm{ccm}$ Wasser $\left(1^{\circ} \mathrm{C}\right)$, die eine Wärmeschuld von 3100 kleinen Kalorien pro kg herstellten, mit einer Herzbeschleunigung, deren Maximum in 13 Minuten erreicht wurde und 33 Schläge pro Minute, nämlich 25\% der Ausgangsfrequenz betrug. 3 Tage nach der Durchtrenung der Splanchnicusnerven wurde der Abkühlungsversuch wiederholt; eine Wassergabe von gleicher Menge und gleicher Temperatur führte auch dabei noch zu einer Frequenzvermehrung des Herzschlages um 30 Schläge pro Minute, nämlich um $27 \%$ in $19 \mathrm{Mi}$ nuten. Während einer kurzen Zeit unmittelbar nach der Wassereinführung war die Pulsfrequenz völlig unverändert oder ein wenig vermindert (nach der Splanchnikotomie), dann kam die frequente Pulsation zutage. Hierbei trat das kontinuierliche, etwa 10 Minuten lang anhaltende Zittern nach etwa 10 Minuten auf; das Zittern schwand vollständig 50 Minuten nach der Wassergabe, statt nach 110 Minuten bei dem Versuch mit intakten Splanchnicusnerven. Die Temperatur sank bei dem zweiten Versuch von $39^{\circ}, 1$ auf $37^{\circ}, 1$ in 25 Minuten, und stieg wieder auf $37^{\circ}, 8$ in 40 Minuten und auf $38^{\circ}, 8$ in 100 Minuten. Die Erniedrigung der Körpertemperatur war etwas kleiner als vorher.

Der Ausgang des Versuches mit einem splanchnikotomierten Hunde stimmte im grossen und ganzen mit denen an demedullierten Hunden so gut wie vollständig überein. Aus diesen Versuchen geht völlig eindeutig hervor, dass das Auftreten der Schlagfrequenzvermehrung des entnervten Herzens bei der Abkühlung durch die Ausschaltung der Epinephrinsekretion gar nicht beeinträchtigt wird. Eine Frequenzvermehrung von einer bestimmten Grösse wird ebenso vor wie nach der Ausschaltung der Epinephrinabsonderung stets durch ein und dieselbe Wärmeschuld hervorgerufen.

Dieser Erfahrung entspricht auch gut das Ergebnis unserer früheren Versuche (W a da, Se o und A b e $)^{205}$ an nicht narkotisierten, nicht gefesselten Hunden, bei denen während der Abkühlung in gleichem oder etwas grösserem Ausmasse, als bei den obenerwähnten Versuchen,

20) Wada, Se o und A be, Tohoku J. of Exp. Med., 1925, 26, 381. 
die in das Nebennierenvenenblut abgesonderte Epinephrinmenge pro Zeiteinheit sehr wenig oder fast nicht vermehrt gefunden war; dabei wurde die erstmalige Entnahme des Nebennierenvenenblutes 13-18 Minuten nach der Kälteapplikation, nämlich im Stadium des kontinuierlichen Zitterns vorgenommen. Bei dem ersten Hunde rief einmal die Einführung einerMenge von kaltem Wasser in den Magen durch eine zuvor angestellte Magenfistel, um eine Wärmeschuld von 3100 kleinen Kalorien pro kg herzustellen, keine erkennbare Zunahme der Sekretionsgeschwindigkeit des Epinephrins hervor; auch 18 Minuten nach der Wassereinführung, d.h. zur Zeit des kontinuierlichen Zitterns blieb die Geschwindigkeit auf dem Anfangswert von 0,00005 mg pro kg pro Minute. Bei dem zweiten Hunde, bei dem eine Wärmeschuld von 3600 kleinen Kalorien vorhanden war, stieg die Sekretionsgeschwindigkeit nur von $0,00003 \mathrm{mg}$ pro $\mathrm{kg}$ pro Minute auf $0,00012 \mathrm{mg} 16$ Minuten nach der Kälteapplikation und näherte sich nach weiteren 20 Minuten wieder dem Ausgangswert. Bei dem dritten Hunde stieg die Geschwindigkeit bei einer Wärmeschuld von 3200 kleinen Kalorien pro $\mathrm{kg}$ von $0,00012 \mathrm{mg}$ pro $\mathrm{kg}$ pro Minute auf $0,00019 \mathrm{mg} 14$ Minuten nach dem Eingeben des Wassers. Bei dem vierten Hunde, bei dem die nach der Wassereinführung hergestellte Wärmeschuld 3400 kleine Kalorien pro $\mathrm{kg}$ betrug, ging die Epinephrinabgabe von $0,00005 \mathrm{mg}$ pro $\mathrm{kg}$ pro Minute auf $0,00008 \mathrm{mg}$ in 13 Minuten und auf $0,00014 \mathrm{mg}$ in 50 Minuten nach der Kälteapplikation.

Dass die unter diesen Umständen abgesonderte Epinephrinmenge nicht genügt, um die Schlagfrequenz des entnervten Hundeherzens in erkennbarer Weise zu vermehren, würde mit der Tatsache in Einklang stehen, dass der pulsfrequenzvermehrende Schwellenwert des Epinephrins an nicht narkotisierten, nicht gefesselten Hunden mit entnervten Herzen in der Nähe von $0,0002-0,0003 \mathrm{mg}$ pro kg pro Minute liegt. ${ }^{21)}$

Deshalb bedarf die Frage, wie die Pulsbeschleunigung bei der Abkühlung zustande kommt, betreffs der herbeigeführten Veränderungen der Blutbeschaffenheit sowie des Kreislaufes einer näheren Untersuchung.

Was ist nun für das Zustandekommen der Beschleunigung des entnervten Herzens durch die Einführung von kaltem Wasser in den Magen verantwortlich zu machen? Es ist wohl nicht zu bezweifeln, dass zu diesem Verhalten der Druck des arteriellen sowie des venö-

21) W a d a und Z. Ka now ok a, Tohoku J. of Exp. Med., 1935, 26, nächstes Heft. 
sen Blutes keinen wesentlichen Anteil hat. ${ }^{22)}$ So verlockend es auch sein mag, die Beschleunigung auf die Sekretion des Sympathins aus den vom sympathischen Nervensystem innervierten Geweben zurückzuführen, so wollen wir uns nicht ohne weiters für diese Annahme entscheiden. Wenn man die aus dem Bostoner Physiologischen Institute hintereinander erschienenen Mitteilungen berücksichtigt, so scheint es berechtigt zu sein, dass eine vermehrte Sekretion des Epinephrins viel bedeutender als die des Sympathins als Agens für die Beschleunigung des entnervten Herzens in Betracht zu ziehen ist. Während die Einführung des kalten Wassers in den Magen beim Hunde keine oder nur eine minimale Hypersekretion des Epinephrins hervorzurufen vermag, wirkt sie auf das entnervte Herz in situ stark beschleunigend ein, und zwar ebenso stark bei Hunden mit intakte wie mit fehlendem Nebennierenmark. Wenn man den Angaben beiptlichten will, dass das Sympathin allein für die Beschleunigung des entnervten Herzens in situ, bei fehlender Epinephrinsekretion verantwortlich zu machen sei, so muss man annehmen, dass das Sympathin in solchen Fällen viel leichter als das Epinephrin aus den Nebennieren sezerniert wird und zwar in so grossen Mengen, dass es genügt das entnervte Herz stark $\mathrm{zu}$ beeinflussen. Nach dieser Überlegung, halte ich es nicht für angebracht, an dieser Stelle in eine weitere Diskussion der Ergebnisse nach den verschiedenen Seiten hin einzutreten.

Es sei bemerkt, dass das Ausmass der bei unseren Hunden ausgeführten Abkühlung annähernd dem bei den Hunden Saitos sowie dem bei den Katzen Ca n nons et al. glich; die bei einem solchen Kälteeinfluss feststellbare Herabsetzung der Körpertemperatur ist eine verhältnismässig geringe und beträgt nicht über $2^{\circ} \mathrm{C}$.

Zum Schluss sei erwähnt, dass die Epinephrinabgabe aus den Nebennieren jedoch durch eine weit stärkere Abkühlung, die eine viel erheblichere Erniedrigung der Körpertemperatur zur Folge hat, gefördert werden kann..$^{0>}$ Bei Hunden, bei denen die Körpertemperatur bis unter $30^{\circ} \mathrm{C}$ herabgesetzt wurde, haben wir eine beträchtliche Epinephrinmehrabgabe bis auf $0,0003-0,0004 \mathrm{mg}$ pro $\mathrm{kg}$ pro Minute beobachtet.

\section{Zusammenfassung.}

Der Einfluss der Einführung von kaltem Wasser in den Magen 406.

22) Siehe diesbezügliche Literatar in Suzuki, Tohoku J. of Exp. Med., 1929, 12, 
auf die Pulszahl von Hunden, bei denen die extrakardialen Herznerven durchschnitten worden waren, wurde vor und nach der Entfernung des Nebennierenmarkes (oder vor und nach der Splanchnikotomie) verglichen. Die dauernde Entnervung wurde durch folgende Eingriffe erreicht: Durch beiderseitige Herausnahme der Ganglia stellata mit den oberen thorakalen sympathischen Grenzsträngen, durch linksseitige Durchtrennung aller vom Vagus sowie vom unteren Halsganglion abzweigenden Äste bis zum unteren Oesophagus und durch rechtsseitige Durchschneidung des Vagosympathicus kurz oberhalb des unteren Halsganglion. Um den Einfluss des herzbeschleunigenden Agens aus der Leber auszuschalten, wurden auch die Lebernerven durchschnitten. Die Kälteapplikation wurde im Ruhezustande der Tiere in der Weise ermöglicht, dass man eine bestimmte Menge kalten Wassers $\left(0^{\circ}, 5-1^{\circ} \mathrm{C}\right)$ mit einem Gummirohr, durch eine vorher angestellte Magenfistel in den Magen einführte. Die hierbei hergestellte Wärmeschuld betrug annähernd 2400-3200 kleine Kalorien pro $\mathrm{kg}$ Körpergewicht.

Bei diesen Hunden bewirkte die Wassereinführung eine beträchtliche Schlagfrequenzsteigerung, die nach 3-7 Minuten auftrat und ihr Maximum nach etwa 15-25 Minuten erreichte. Die Schlagfrequenz stieg maximal um 19-41 Schläge pro Minute, nämlich um 12-39\% der Ausgangsfrequenz. Meist beharrte die Schlagzahl während einiger Minuten auf dem höchsten Wert, um dann abzusteigen und 1-2 Stunden nach der Kälteapplikation ihre Ausgangszahl wieder zu erreichen. Die maximale Herzbeschleunigung wurde gewöhnlich zur Zeit des nach der Kälteapplikation am stärksten auftretenden Zitterns oder ein wenig später festgestellt. Diese Schlagfrequenzsteigerung bei der Abkühlung trat nach der Ausschaltung des Epinephrineinflusses in demselben Ausmasse und derselben Ausdauer in Erscheinung, wie vorher; in dieser Tatsache finden wir einen Hinweis darauf, dass diese Frequenzvermehrung nicht durch die Epinephrinabsonderung aus den Nebennieren bedingtist. Dies steht mit unseren früheren Versuchen an nicht narkotisierten, nicht gefesselten Hunden im besten Einklang, bei denen eine Abkühlung in gleichem oder etwas grösserem Ausmasse als bei den eben beschriebenen Versuchen vorgenommen worden war, die aber keine erkennbare vermehrte Epinephrinsekretion gezeigt hatten.

Die vorliegende Arbeit wurde mit gütiger Unterstützung von Herrn Dr. Z. Ka no woka ausgeführt, wofür ich ihm zu grösstem Dank verpflichtet bin. 